The Right of Publicity 



\section{The Right of Publicity}

Privacy Reimagined for a Public World

Jennifer E. Rothman

$\sum_{m}^{m}$ 
Copyright () 2018 by Jennifer E. Rothman

All rights reserved

Printed in the United States of America

\section{First printing}

\section{Library of Congress Cataloging-in-Publication Data}

Names: Rothman, Jennifer E., 1969- author.

Title: The right of publicity : privacy reimagined for a public world / Jennifer E. Rothman.

Description: Cambridge, Massachusetts : Harvard University Press, 2018. |

Includes bibliographical references and index.

Identifiers: LCCN 2017045240 | ISBN 9780674980983 (alk. paper)

Subjects: LCSH: Publicity (Law) - United States. | Privacy, Right of-United States. |

Personality (Law) - United States. Intellectual property-United States.

Classification: LCC KF1262 .R68 2018 | DDC 342.7308/58-dc23

LC record available at https://lccn.loc.gov/2017045240

Cover photo: Meinzahn/iStock/Thinkstock/Getty Images

Cover design: Jill Breitbarth 
For Elijah and Hazel 
